\title{
Femtonewton Force Sensing with Optically Trapped Nanotubes
}

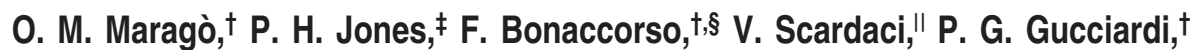 \\ A. G. Rozhin," and A. C. Ferrari ${ }^{\star, \|}$
}

CNR-Istituto per i Processi Chimico-Fisici, Salita Sperone C.da Papardo, I-98158 Messina, Italy, Department of Physics and Astronomy, University College

London, Gower Street, London WClE 6BT, U.K., Dipartimento di Fisica della Materia

e Ingegneria Elettronica, Universitá di Messina, Salita Sperone 31, I-98166 Messina,

Italy, and Engineering Department, University of Cambridge, 9 JJ Thomson Avenue,

Cambridge CB3 OFA, U.K.

Received May 29, 2008; Revised Manuscript Received August 5, 2008

\begin{abstract}
We extract the distribution of both center-of-mass and angular fluctuations from three-dimensional tracking of optically trapped nanotubes. We measure the optical force and torque constants from autocorrelation and cross-correlation of the tracking signals. This allows us to isolate the angular Brownian motion. We demonstrate that nanotubes enable nanometer spatial and femtonewton force resolution in photonic force microscopy, the smallest to date. This has wide implications in nanotechnology, biotechnology, nanofluidics, and material science.
\end{abstract}

Optical tweezers ${ }^{1,2}$ are versatile tools to manipulate matter from the micrometer to the atomic scale and have many applications in biology and physics. ${ }^{3}$ Their soft touch can hold and manipulate a single atom ${ }^{4}$ as well as individual cells and intracellular matter in vivo. ${ }^{5}$ Optical trapping ${ }^{1,2}$ of one-dimensional nanostructures has received much attention due to its potential for top-down organization of complex nanoassemblies. ${ }^{6-10}$ Trapped nanoparticles can also be used to increase space and force resolution ${ }^{11}$ in photonic force microscopy. ${ }^{12}$ Their small transverse size is the key to achieve nanometric resolution, while an axial dimension in the micrometer range ensures stable trapping and could allow force sensing in the subpiconewton regime.

Brownian motion, ${ }^{13}$ i.e., the continuous random displacements of small particles in a fluid, is a fundamental ingredient in force sensing with optical tweezers. ${ }^{12}$ For an optically trapped particle, a statistical analysis of the fluctuations about the equilibrium position enables the measurement of piconewton forces. ${ }^{14}$ In order to reach the ultimate space and force resolution, it is necessary to get a full understanding of the Brownian dynamics of extended nonspherical objects in the optical trap. In particular, the effects of the external hydrodynamic environment leading to Brownian fluctuations in both position and orientation ${ }^{15}$ define the ultimate resolution limits. However, it is only recently that direct visualiza-

\footnotetext{
* Corresponding author. E-mail: acf26@eng.cam.ac.uk.

$\dagger$ CNR-Istituto per i Processi Chimico-Fisici.

* University College London.

§ Universitá di Messina.

"University of Cambridge.
}

tion of rotational Brownian motion (i.e., continuous random orientational fluctuations), and its coupling with translational degrees of freedom, was demonstrated for microellipsoids $(3.3 \times 0.6 \mu \mathrm{m}$ size $)$ confined in two dimensions. ${ }^{15}$ Here we push this to the nanoscale by tracking the Brownian motion of nanotubes.

Samples are prepared using purified HiPCO single-wall nanotubes (SWNT) (Carbon Nanotechnology Inc.) dispersed in water with sodium dodecyl benzene sulfonate. ${ }^{16,17}$ Solutions are ultrasonicated and filtered to ensure removal of residual catalyst particles, amorphous carbon, and big bundles. ${ }^{18} \mathrm{~A}$ few tens microliters are placed in a $100 \mathrm{~mm}^{3}$ chamber attached to a piezo-stage with $1 \mathrm{~nm}$ resolution. Optical trapping is done by tightly focusing a $830 \mathrm{~nm}$ laser beam through a $100 \times$ oil immersion objective $(\mathrm{NA}=1.3)$ on an inverted microscope (see Figure 1a). Positional and angular displacements are detected using back focal plane (BFP) interferometry. ${ }^{19,20}$ The interference pattern between forward scattered and unscattered light in the microscope condenser BFP is imaged onto a four quadrant photodiode (QPD), which is oriented with the polarization $(x)$ axis of the laser beam in order to have sensitivity over polarization. ${ }^{21}$ An analog circuit board combines the signals as pairwise sums $\left(S_{x}, S_{y}\right)$ and four-quadrant sum $\left(S_{z}\right)$. For spherical objects, the three signals $\left(S_{x}, S_{y}, S_{z}\right)$ are proportional to the center-of-mass displacements, while for nonspherical they also contain angular information, ${ }^{22}$ since fluctuations in azimuthal and polar angles change the interference pattern. 


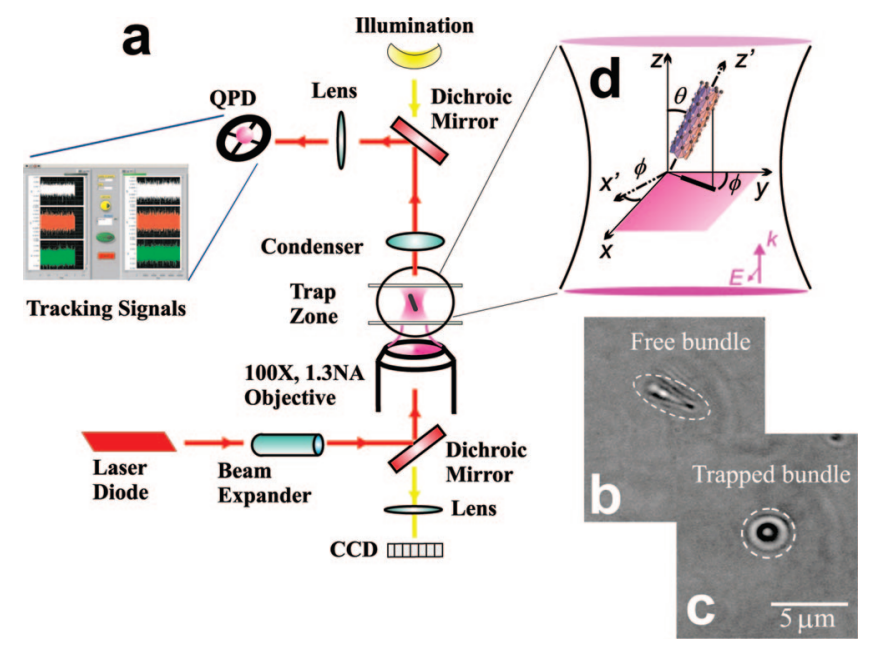

Figure 1. Optical trapping setup and geometry. (a) A laser beam is expanded to overfill the back aperture of a high numerical aperture lens. The light is focused in a chamber, with the SWNT solution. A CCD is used to image the sample. A quadrant photodiode (QPD) detects the interference from the back focal plane of the condenser that collects the light from the trapping region. (b) Image of an untrapped bundle. (c) Image of the same bundle trapped and oriented by radiation torque along the optical axis. (d) Geometry, with relevant Euler angles and variables.

The particles are imaged through the same objective onto a charge coupled device (CCD) camera, with diffraction limited resolution. Panels $\mathrm{b}$ and $\mathrm{c}$ of Figure 1 show a SWNT bundle with a length of about $3 \mu \mathrm{m}$. In the first image (Figure 1b) the untrapped bundle is free floating and randomly oriented (see video in Supporting Information), while Figure $1 \mathrm{c}$ has the optically trapped structure aligned with the laser propagation direction (the imaging axis). The CCD camera acquisition is slow (25 frames/s) and can only detect lowfrequency Brownian fluctuations for untrapped particles. Thus, we rely upon BFP interferometry and QPD detection, enabling a much higher sampling rate $(50 \mathrm{kHz})$, in order to measure the Brownian motion for trapped particles.

In general, the orientation of a linear nanostructure in an optical trap is related to its length. Long nanostructures, as in our case, are aligned by the radiation torque along the propagation axis. ${ }^{23}$ Here we measure both translational and angular fluctuations around this equilibrium position.

The geometry of our experiment is shown in Figure 1d. The laser beam propagates along $z$ with initial polarization along $x$. We consider the Euler angles $(\phi, \theta, \psi)$ of the SWNT bundle with respect to the laboratory axes $x y z$. For an axially symmetric object we can always choose the third angle $\psi$ to be 0 . For small displacements from equilibrium, the singlebeam optical trap is well approximated by a harmonic potential

$$
V\left(x_{i}\right)=\frac{1}{2} \sum_{i=x, y, z} k_{i} x_{i}^{2}
$$

with spring constants $k_{z}<k_{x}, k_{y}{ }^{24}$ These depend on both the geometry of the trapped particle and the parameters of the propagating focused Gaussian beam, such as power and polarization. $^{21}$

Figure 2 visualizes the Brownian motion of a trapped SWNT bundle, reconstructed from the photodiode signals, compared with the motion of a trapped $2 \mu \mathrm{m}$ latex sphere in Figure $2 b$, both measured in the same experimental conditions of laser power and temperature $\left(24^{\circ} \mathrm{C}\right)$. The data-points are extracted from the $S_{i}(t)$ signals acquired for $2 \mathrm{~s}$ at 50 $\mathrm{kHz}$ sampling rate ( $10^{5}$ points per channel). The high aspect ratio of longitudinal $\left(S_{z}\right)$ and transverse $\left(S_{x}, S_{y}\right)$ motion for the nanotubes (Figure 2, panels a, c, d, and e) shows an increase in mobility with respect to the spherical particle (Figure 2b). In order to understand this behavior, we now model the optical trapping of SWNTs.

For rigid rodlike structures, such as a SWNT bundle, the viscous drag is described by an anisotropic hydrodynamic mobility tensor, ${ }^{25}$ the components of which depend on the length-to-diameter ratio $p=L / d$ as $^{26}$ (see also Supporting Information)

$$
\begin{gathered}
\Gamma_{\perp}=\frac{\ln p+\delta_{\perp}}{4 \pi \eta L}, \quad \Gamma_{\|}=\frac{\ln p+\delta_{\|}}{2 \pi \eta L} \\
\Gamma_{\Theta}=\frac{3\left(\ln p+\delta_{\theta}\right)}{\pi \eta L^{3}}
\end{gathered}
$$

where $\Gamma_{\perp}$ and $\Gamma_{\|}$are the translational mobilities, transverse and parallel to the axis, $\Gamma_{\Theta}$ is the rotational mobility about midpoint, $\eta$ is the water dynamical viscosity, and $\delta_{i}$ are endcorrections, calculated by ref 26 as polynomial of $(\ln 2 p)^{-1}$.

The projections onto the laboratory axes are determined through the relevant Euler angles $(\phi, \theta)$ and the corresponding rotation matrix. ${ }^{27}$ Since the trapped bundle is aligned with $z$, fluctuations occur in the small polar angle limit, $\theta \ll 1$, and the QPD signals are (see Supporting Information)

$$
\begin{gathered}
S_{x} \sim \beta_{x}\left(X+L \Theta_{x}\right) \\
S_{y} \sim \beta_{y}\left(Y+L \Theta_{y}\right) \\
S_{z} \sim \beta_{z} Z
\end{gathered}
$$

where $\beta_{i}$ values are the detector calibration factors, $X, Y$, and $Z$ are the center-of-mass coordinates, $L$ is the bundle length, and $\Theta_{x}=\theta \sin \phi$ and $\Theta_{y}=\theta \cos \phi$ are the projections on the $x$ and $y$ axis, respectively (shown in Figure 1d). $S_{z}$ is thus not much affected by the angular motion, having a cos $\theta$ dependence only.

We now treat the center-of-mass $X_{i}$ and angular $\Theta_{j}$ coordinates as stochastic variables. Thus, we describe the Brownian dynamics of our trapped nanotubes by a set of uncoupled Langevin equations ${ }^{28}$

$$
\begin{gathered}
\partial_{t} X_{i}(t)=-\omega_{i} X_{i}(t)+\xi_{i}(t), \quad i=x, y, z \\
\partial_{t} \Theta_{j}(t)=-\Omega_{j} \Theta_{j}(t)+\xi_{j}(t), \quad j=x, y
\end{gathered}
$$

where $\omega_{i}=\Gamma_{i} k_{i}$ and $\Omega_{j}=\Gamma_{\Theta} k_{j}$ are relaxation frequencies, related to the force and torque constants and mobility tensor components, while $\xi_{i}(t)$ are random noise sources with zero mean and variance $\left\langle\xi_{i}(t) \xi_{i}(t+\tau)\right\rangle=2 k_{\mathrm{B}} T \Gamma_{i} \delta(\tau)$.

The torque of the confining potential about the laboratory axes is only relevant for the orientational dynamics, while not affecting the center-of-mass motion in a small angle regime. Also, due to the strong $z$-alignment and because angular fluctuations are small, the radiation torque along $z$ is small. Thus, possible precessional motion about $z$ is slow on the time scale of all other motions and has small amplitude (see Supporting Information). We then neglect all coupling 

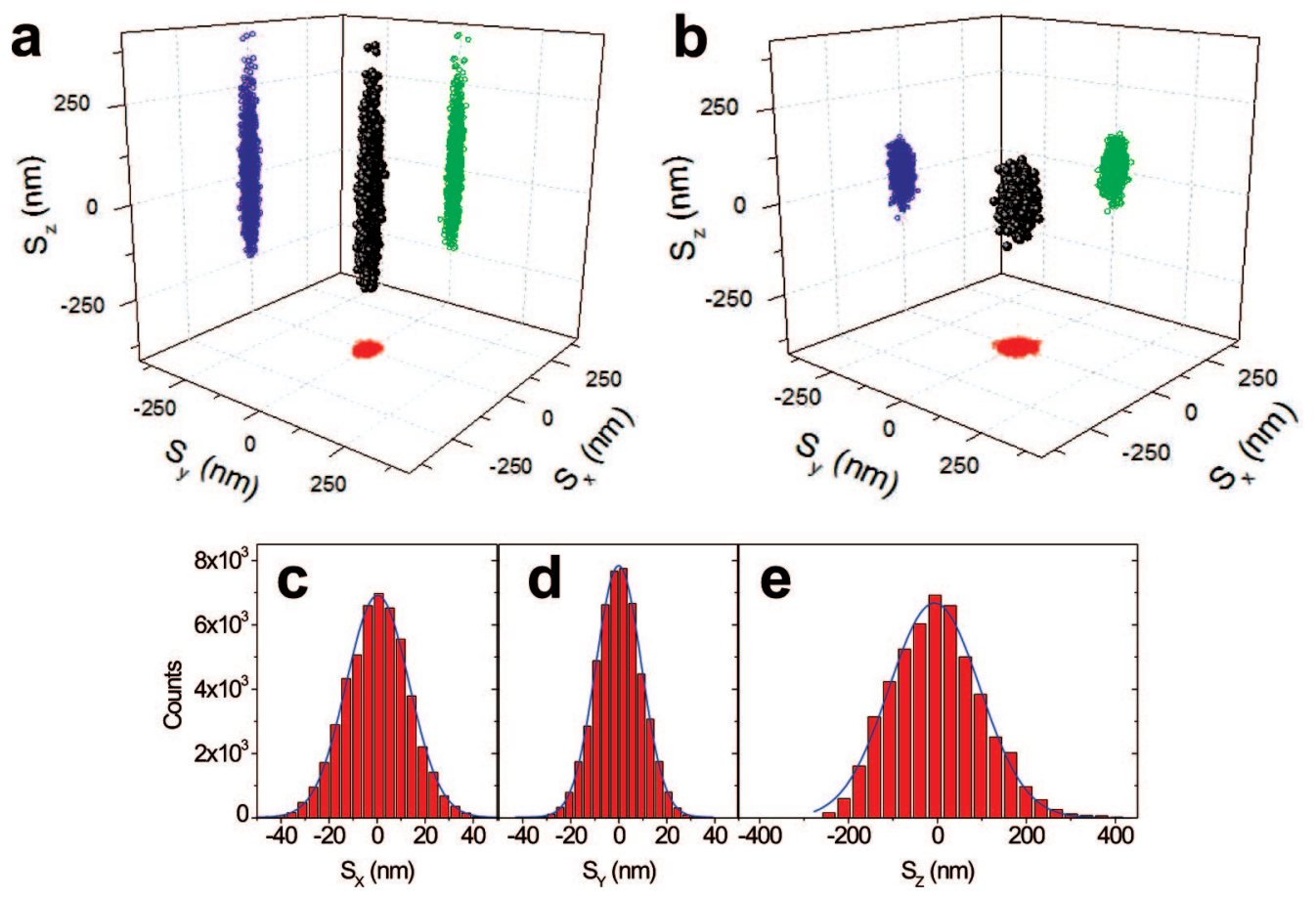

Figure 2. Brownian motion in the optical trap. (a) Trapped SWNT bundle and (b) $2 \mu \mathrm{m}$ latex bead. In both cases the tracking (black dots) is obtained by extracting 2000 points from the QPD signals $S_{i}(t)$. The projections of the motion in $x y$ (red dots), $x z$ (blue dots), and $y z$ (green dots) are also shown. For the trapped nanotubes, an increase in mobility compared to the spherical particle along the $z$ axis is evident. (c, d) Histograms of the transverse signals $S_{x}(t), S_{y}(t)$ obtained from the full record of $10^{5}$ points. In the transverse direction, both translational and angular fluctuations are convoluted into a signal that includes both center of mass displacement and the projection of the bundle onto the measurement axis. The difference in the root-mean-square widths of the fluctuations in $x$ and $y$ arises from the different curvatures of the optical potential in the directions parallel and perpendicular to the initial polarization to which the SWNT bundle is sensitive. For each graph the QPD voltage-to-position calibration factors $\beta_{i}$ are obtained using the calculated mobility coefficients and amplitude of the signals' autocorrelation functions $C_{i i}(0)=\beta_{i}{ }^{2} k_{\mathrm{B}} T / k_{i}$ for the position fluctuation contributions only. The root mean squares of the transverse displacements, extracted from a Gaussian fit, are $\left\langle x^{2}\right\rangle^{1 / 2}=13 \mathrm{~nm}$ and $\left\langle y^{2}\right\rangle^{1 / 2}=9 \mathrm{~nm}$. (e) Histogram of the longitudinal signal $S_{z}(t)$. This is only due to fluctuations of the center of mass and has a measured root mean square of $\left\langle z^{2}\right\rangle^{1 / 2}=100 \mathrm{~nm}$.

terms in the Langevin equations. As shown later, this is also validated by our experimental observations.

Tracking signals are studied by correlation analysis of the Brownian fluctuations. ${ }^{29}$ For spherical particles, these show a single exponential decay at the related Langevin equation relaxation frequency. ${ }^{30}$ From eqs 3,4 , and 5, the autocorrelations of the transverse QPD signals $C_{i i}(\tau)=\left\langle S_{i}(t) S_{i}(t+\right.$ $\tau)\rangle$ now contain combined information on center-of-mass and angular fluctuations, and decay with lag time $\tau$ as a doubleexponential with positional and angular relaxation frequencies $\omega_{i}, \Omega_{i}(i=x, y)$. Furthermore, as the angular variables are geometrically correlated $\left(\Theta_{x}=\Theta_{y} \tan \phi\right)$, the crosscorrelations $C_{x y}(\tau)=\left\langle S_{x}(t) S_{y}(t+\tau)\right\rangle=C_{y x}(-\tau)$ of the transverse signals decay as single exponentials with relaxation rates different for positive and negative lag times, corresponding to $\Omega_{x}$ and $\Omega_{y}$ (see Supporting Information).

For our trapped bundles, we measure $L$ from CCD images, such as those in Figure 1b,c $(L \approx 3 \mu \mathrm{m})$. A direct estimate of the bundles transverse size is obtained from atomic force microscopy (AFM) measurements on a dried drop of solution. These give an average transverse size of $\sim 11 \mathrm{~nm}$. Note that even an uncertainty of $50 \%$ in the transverse size would yield only a $6 \%$ error on the mobility parameters, due to their logarithmic dependence on $p$. Thus, using $d=11$ $\mathrm{nm}, L=3 \mu \mathrm{m}$ and inserting $p=L / d=273, \eta\left(24^{\circ} \mathrm{C}\right)=$ $0.911 \mathrm{mPa}$ s in eqs 1 and 2 , we obtain numerical values for the mobility parameters: $\Gamma_{\perp} \sim 184 \mu \mathrm{m} /(\mathrm{pN} \mathrm{s}), \Gamma_{\|} \sim 307$ $\mu \mathrm{m} /(\mathrm{pN} \mathrm{s}), \Gamma_{\Theta} \sim 192(\mu \mathrm{m} \mathrm{pN} \mathrm{s})^{-1}$.

These allow us to derive the optical force constants from the relaxation frequency measurements, as follows. Figure 3 shows a representative correlation function analysis of the tracking signals $S_{i}(t)$. Panels a, b, and c of Figure 3 are plotted on a linear lag time scale and indicate that the correlation functions have a high-amplitude fast dynamics (kHz range) and small-amplitude slow oscillations $(\sim 5 \mathrm{~Hz})$, similar to what recently observed for optically trapped beads. ${ }^{30}$ This is typical of a rotational motion about the $z$-axis, i.e., in the case of our nanotubes, a slow small amplitude precession, and again justifies our assumption that precessional motion in the trap is slow compared to other motions, and may be neglected. The fast features are instead related to the Brownian motion of the center of mass and angular degrees of freedom, plotted for greater clarity on a logarithmic scale in Figure 3, panels d, e, and $\mathrm{f}$. The autocorrelation functions of the transverse signals, $C_{x x}$ and $C_{y y}$, are shown in Figure $3 \mathrm{~d}$ (circles), and are well fitted by two exponentials (solid lines; dotted lines correspond to the best single exponential fit) with decay rates of $\omega_{x}=(4.1 \pm 0.2) \times 10^{3} \mathrm{~s}^{-1}, \Omega_{x}=$ $(0.79 \pm 0.08) \times 10^{3} \mathrm{~s}^{-1}$ and $\omega_{y}=(10.2 \pm 0.8) \times 10^{3} \mathrm{~s}^{-1}$, $\Omega_{y}=(1.26 \pm 0.05) \times 10^{3} \mathrm{~s}^{-1}$. Figure $3 \mathrm{f}$ indicates that $C_{z z}$ fits to a single exponential decay with rate $\omega_{z}=(0.26 \pm$ $0.03) \times 10^{3} \mathrm{~s}^{-1}$ and, thus, is not affected by angular 


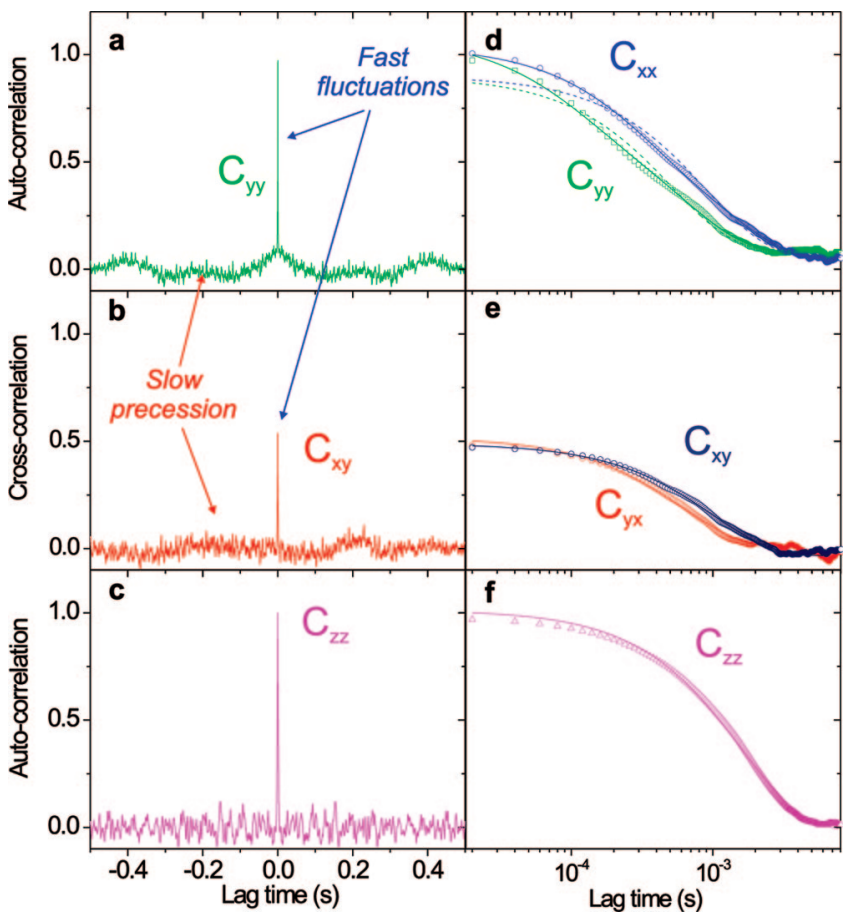

Figure 3. Correlation analysis. (a) Transverse signal autocorrelation and (b) signal cross-correlation on a linear scale for lag time. The separation of time scales for fast center-of-mass and polar angle fluctuations, and slow azimuthal precession is evident.(c) Longitudinal signal autocorrelation on linear scale. Only a fast centerof-mass fluctuations signal is seen.(d) Signal autocorrelation and (e), signal cross-correlation, plotted on a logarithmic scale. Solid lines show a double-exponential fit for the autocorrelation of the transverse signals and a single exponential for the cross-correlation with a decay rate consistent with that found in the autocorrelation. (f) Autocorrelation of longitudinal signal on logarithmic time scale, showing a single exponential fit, with much longer decay time than for transverse signals.

fluctuations. The cross-correlations of the transverse signals $C_{x y}$ and $C_{y x}$ shown in Figure 3e (for positive lag time only) are fitted by a single exponential with decay rate $\Omega_{y}=(1.31$ $\pm 0.03) \times 10^{3} \mathrm{~s}^{-1}$ and $\Omega_{x}=(0.85 \pm 0.02) \times 10^{3} \mathrm{~s}^{-1}$, respectively, that are consistent with the values obtained from the autocorrelation functions. From these measurements, and the mobility parameters calculated above, we obtain the spring constants $k_{i}(i=x, y, z)$ and torque constants $k_{\Theta_{i}}(i=$ $x, y)$ plotted in Figure 4(a,b). This shows that the optical trapping parameters scale linearly with laser power.

Another fundamental aspect of laser trapping is light polarization. Its effects on trapped submicrometer particles yield different spring constants in the $x y$ plane of the trapping potential. ${ }^{24}$ This asymmetry can be quantified using the parameter $k_{P}=1-k_{x} / k_{y}{ }^{21,23}$ From Figure $4 \mathrm{c}$, we obtain an average value $k_{P} \approx 0.53$ for our SWNT bundles, which is consistent with the calculated value for spherical particles much smaller than the trapping wavelength. ${ }^{21}$ This demonstrates that polarization effects are important also for extended nanostructures and depend only on the nanometric transverse size, and not the length. Finally, to explain the large Brownian motion asymmetry of Figure 2a, we plot in Figure $4 \mathrm{~d}$ a parameter which quantifies the trap aspect ratio: $\left(k_{x}+k_{y}\right) / 2 k_{z}$. Due to the equipartition theorem, ${ }^{28}$ the variances of translational displacements (giving the widths of the histograms in Figure 2, panels c, d, and e) are linked to the force constants $\left\langle x_{i}^{2}(t)\right\rangle=k_{\mathrm{B}} T / k_{i}$. From our data, this parameter is $\sim 45$, while for the latex spherical particle of Figure $2 b$ it is $\sim 5$. This significant difference shows how the optical confining potential strongly depends on the geometry of the trapped particle.

The minimum laser power for which we can stably trap our SWNT bundles is $\sim 2 \mathrm{~mW}$. This corresponds (see also Figure $4 \mathrm{a}$ ) to force constants of $k_{x} \approx 2.1 \mathrm{pN} / \mu \mathrm{m}, k_{y} \approx 2.8$ $\mathrm{pN} / \mu \mathrm{m}$ in the transverse plane, and $k_{z} \approx 20 \mathrm{fN} / \mu \mathrm{m}$ on the optical axis. These values, together with Brownian fluctuations, determine the force sensing and spatial resolution in a photonic force microscopy experiment. We can give a conservative estimate of our force resolution $\Delta F_{z}$ as an external force shifting the average axial position in the trap (i.e., the direction affected when probing a surface) by an amount equal to the root-mean-square of the fluctuations, i.e., $\Delta F_{z} \sim k_{z}\left\langle z^{2}\right\rangle^{1 / 2}$. In our setup, at the minimum power required for stable trapping, we measure $\left\langle z^{2}\right\rangle_{\min }^{1 / 2} \approx 0.4 \mu \mathrm{m}$, giving a force resolution $\Delta F_{z} \approx 8 \mathrm{fN}$. This femtonewton force resolution in the axial direction is achieved together with a transverse spatial resolution of $\left\langle x^{2}\right\rangle_{\min }{ }^{1 / 2} \approx 42 \mathrm{~nm}$, as measured from the root mean square of the transverse fluctuations. Similarly, we can estimate the resolution for our maximum power (limited to $\sim 17 \mathrm{~mW}$ by our diode laser), where we have $k_{z} \approx 700 \mathrm{fN} / \mu \mathrm{m}$ and $\left\langle z^{2}\right\rangle_{\max }{ }^{1 / 2} \approx 0.1$ $\mu \mathrm{m}$. Thus, the force-sensing resolution at maximum power is $\Delta F_{z} \approx 70 \mathrm{fN}$, with a transverse spatial resolution of $\sim 10$ $\mathrm{nm}$. Note that increasing the laser power creates a stiffer trap, worsening the force sensing resolution. On the other hand, the spatial resolution becomes better at higher power because the variance of the Brownian fluctuations is inversely proportional to the trap stiffness $\left\langle x_{i}^{2}\right\rangle=k_{\mathrm{B}} T / k_{i}$. Thus, low power seems better suited for force-sensing applications, although, in general, a compromise has to be achieved depending on the probe-sample interaction and sizes. For spherical particles, while force sensing in the subpiconewton regime was reported, ${ }^{14,24}$ the spatial resolution is strongly limited by the bead diameter. ${ }^{12}$ For example, from the measurements on the $2 \mu \mathrm{m}$ bead shown in Figure $2 \mathrm{~b}$, we estimate a force sensing resolution of about $300 \mathrm{fN}$, with a spatial resolution limited by the $2 \mu \mathrm{m}$ bead size, and not the $100 \mathrm{~nm}$ width of the Brownian center of mass fluctuations. This holds until the bead size is larger than the Brownian fluctuations. Trapping spherical nanoparticles smaller than $100 \mathrm{~nm}$ is difficult, since the radiation force scales with volume, so that trapping is overcome by Brownian fluctuations. ${ }^{3}$ Although metal nanoparticles as small as $20 \mathrm{~nm}$ were trapped exploiting their plasmon resonances, ${ }^{31}$ their stable 3D trapping is still difficult ${ }^{32}$ and is accompanied by heating, ${ }^{33}$ limiting their use in temperature-sensitive experiments or when robust trapping over long time is needed (e.g., during a surface scan). Indeed, the crucial role of nanotubes in femtonewton force sensing is the combination of their small transverse size and their uniaxial anisotropy. This leads to stable trapping even at very low power $(1-2 \mathrm{~mW})$, thus allowing femtonewton force sensing, while keeping the 


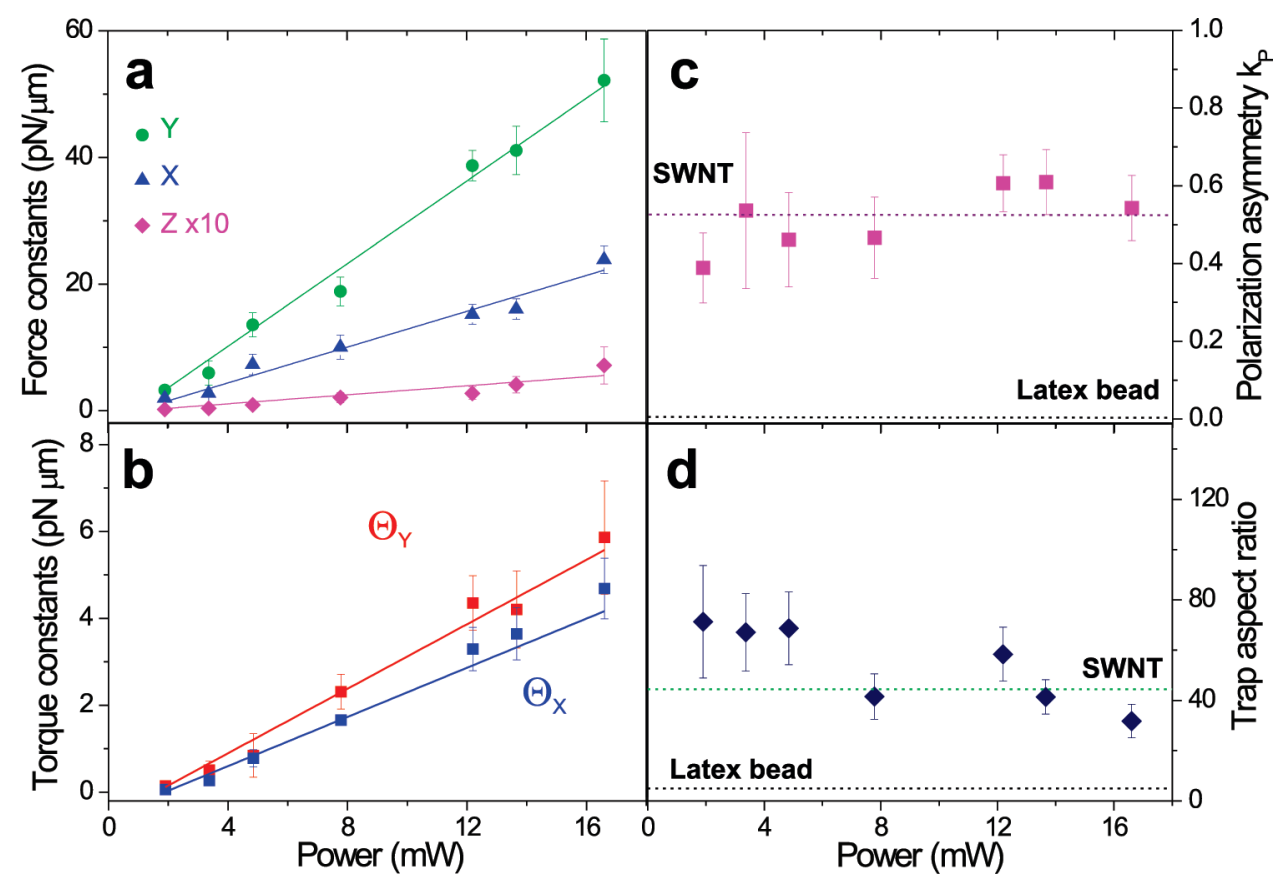

Figure 4. Optical trapping parameters as a function of laser power. (a) Spring constants $k_{i}(i=x, y, z)$. (b) Torque constants about the transverse axes, $k_{\Theta_{i}}(i=x, y)$. (c) Polarization asymmetry in the transverse plane $k_{p}$. The average value (magenta dashed line) of $k_{p} \approx 0.53$ for nanotubes is consistent with calculations for subwavelength particles. The average value for a $2 \mu \mathrm{m}$ latex bead (black dashed line) is $k_{p} \approx 0.07$. (d) Trap aspect ratio, with average an order of magnitude larger for the SWNT bundles (green dashed line) than for spherical particles (black dashed line). Data in all plots are averages over four measurements and error bars represent the shot-to-shot fluctuations from the mean value.

transverse resolution within tens of nanometers. Contrary to the micrometer sized beads, the spatial resolution for such nanostructures is limited by the Brownian fluctuations. Clearly, our novel technique can be extended to other uniaxial nanostructures, such as nanowires, which can also be optically trapped. ${ }^{10,11}$

In conclusion, we revealed the angular fluctuations for optically trapped nanotube bundles, and elucidated some of the complex dynamics and hydrodynamics of these quasione-dimensional objects. We demonstrated how to separate the center-of-mass and angular contributions to the tracking signals by a correlation function analysis and thus measured both the optical force and torque constants. Our ability to discriminate linear and angular motion is of great importance for fundamental understanding of the optical trapping mechanisms and for applications of optically trapped nanotubes in photonic force microscopy. Our measurements show that force sensing as low as $8 \mathrm{fN}$ with a transverse spatial resolution of about $40 \mathrm{~nm}$ is possible, far surpassing any previous result based on latex spheres.

Acknowledgment. We are grateful to F. Borghese, N. Lo Iudice, F. Palmisano, and G. Calogero for fruitful discussions. We acknowledge support from the Royal Society and British Council. V.S., A.G.R., and A.C.F. acknowledge funding from EPSRC Grants GR/S97613/01 and EP/E500935/1. A.C.F. acknowledges funding from the Royal Society, the Leverhulme and Newton Trusts.

Supporting Information Available: Descriptions of hydrodynamics of a carbon nanotube, the torque and Lan- gevin equations, and correlation function of the quadrant photodiode signals and a video showing how a bundle is trapped and aligned. This material is available free of charge via the Internet at http://pubs.acs.org.

\section{References}

(1) Ashkin, A. Acceleration and Trapping of Particles by Radiation Pressure. Phys. Rev. Lett. 1970, 24, 156-159.

(2) Ashkin, A.; Dziedzic, J. M.; Bjorkholm, J.E.; Chu, S. Observation of a single-beam gradient force optical trap for dielectric particles. Opt. Lett. 1986, 11, 288.

(3) Ashkin, A. Optical Trapping and Manipulation of Neutral Particles using Lasers; World Scientific Publishing: Singapore, 2007, and references therein.

(4) Miroshnychenko, Y.; Alt, W.; Dotsenko, I.; Farster, L.; Khudaverdyan, M.; Meschede, D.; Schrader, D.; Rauschenbeutel, A. Quantum engineering: An atom-sorting machine. Nature 2006, 442, 151.

(5) Svoboda, K.; Block, S. M. Biological applications of optical forces. Annu. Rev. Phys. Biomol. Struct. 1994, 23, 247-285.

(6) Tan, S.; Lopez, H. A.; Cai, C. W.; Zhang, Y. Optical trapping of singlewalled carbon nanotubes. Nano Lett. 2004, 4, 1415-1419.

(7) Plewa, J.; Tanner, E.; Mueth, D.; Grier, D. Processing carbon nanotubes with holographic optical tweezers. Opt. Express 2004, 12, 1978.

(8) Agarwal, R.; Ladavac, K.; Roichman, Y.; Yu, G.; Lieber, C.; Grier, D. Manipulation and assembly of nanowires with holographic optical traps. Opt. Express 2005, 13, 8906-8912.

(9) Zhang, J.; Kim, H. I.; Oh, C. H.; Sun, X.; Lee, H. Multidimensional manipulation of carbon nanotube bundles with optical tweezers. Appl. Phys. Lett. 2006, 88, 053123.

(10) Pauzauskie, P. J.; Radenovic, A.; Trepagnier, E.; Shroff, H.; Yang, P.; Liphardt, J. Optical trapping and integration of semiconductor nanowire assemblies in water. Nat. Mater. 2006, 5, 97-111.

(11) Nakayama, Y.; Pauzauskie, P. J.; Radenovic, A.; Onorato, R. M.; Saykally, R. J.; Liphardt, J.; Yang, P. Tunable nanowire nonlinear optical probe. Nature 2007, 447, 1098-1102.

(12) Florin, E.-L.; Pralle, A.; Horber, J. K. H.; Stelzer, E. H. K. Photonic force microscope based on optical tweezers and two-photon excitation for biological applications. J. Struct. Biol. 1997, 119, 202-211. 
(13) Einstein, A. Über die von der molekularkinetischen Theorie der Wärme geforderte Bewegung von in ruhenden Flüssigkeiten suspendierten Teilchen. Ann. Phys. 1905, 17, 549-560.

(14) Smith, D. E.; Tans, S. J.; Smith, S. B.; Grimes, S.; Anderson, D. L.; Bustamante, C. The bacteriophage phi 29 portal motor can package DNA against a large internal force. Nature 2001, 413, 748-752.

(15) Han, Y.; Alsayed, A. M.; Nobili, M.; Zhang, J.; Lubensky, T. C.; Yodh, A. G. Brownian motion of an ellipsoid. Science 2006, 314, 626-630.

(16) Islam, M. F.; Rojas, E.; Bergey, D. M.; Johnson, A. T.; Yodh, A. G. High Weight Fraction Surfactant Solubilization of Single-Wall Carbon Nanotubes in water. Nano Lett. 2003, 3, 269.

(17) Moore, V. C.; Strano, M. S.; Haroz, E. H.; Hauge, R. H.; Smalley, R. E.; Schmidt, J.; Talmon, Y. Individually Suspended Single-Walled Nanotubes in various surfactants. Nano Lett. 2003, 3, 1379-1382.

(18) Scardaci, V.; Rozhin, A. G.; Hennrich, F.; Milne, W. I.; Ferrari, A. C Carbon Nanotubes-polymer composites for photonic devices. Physica E 2007, 37, 115-118.

(19) Gittes, F.; Schmidt, C. F. Interference model for back-focal-plane displacement detection in optical tweezers. Opt. Lett. 1998, 23, 7-9.

(20) Pralle, A.; Prummer, M.; Florin, E.-L.; Stelzeer, E. H. K.; Hörber, J. K. H. Three-Dimensional High-Resolution Particle Tracking for Optical Tweezers by Forward Scattered Light. Microsc. Res. Tech. 1999, 44, 378-386.

(21) Borghese, F.; Denti, P.; Saija, R.; Iatí, M. A. Optical trapping of nonspherical particles in the T-matrix formalism. Opt. Express 2007, 15, 11984-11998.

(22) Kress, H.; Stelzer, E. H.; Rohrbach, A. Tilt angle dependent threedimensional-position detection of a trapped cylindrical particle in a focused laser beam. Appl. Phys. Lett. 2004, 84, 4271-4273.
(23) Borghese, F.; Denti, P.; Saija, R.; Iatí, M. A.; Maragò, O. M. Radiation force and torque on optically trapped linear nanostructures. Phys. Rev. Lett. 2008, 100, 163903.

(24) Rohrbach, A. Stiffness of optical traps: Quantitative agreement between experiment and electromagnetic theory. Phys. Rev. Lett. 2005, 95, 168102.

(25) Happel, J.; Brenner, H. Low Reynolds Number Hydrodynamics; Springer: Berlin, 1981.

(26) Broersma, S. Viscous force and torque constants for a cylinder. J. Chem. Phys. 1981, 74, 6989.

(27) Goldstein, H.; Poole, C. P.; Safko, J. L. Classical Mechanics, 3rd ed., 3rd ed.; Addison Wesley: Boston, MA, 2001.

(28) Coffey, W. T., Kalmykov, Y. P., Waldron, J. T. The Langevin Equation, 2nd ed.; World Scientific: Singapore, 2004.

(29) Martin, S.; Reichert, M.; Stark, H.; Gisler, T. Direct Observation of Hydrodynamic Rotation-Translation Coupling between Two Colloidal Spheres. Phys. Rev. Lett. 2006, 97, 248301.

(30) Volpe, G.; Petrov, D. Torque detection using brownian fluctuations. Phys. Rev. Lett. 2006, 97, 210603.

(31) Hansen, P. M.; Bhatia, V. K.; Harrit, N. \&; Oddershede, L. Expanding the Optical Trapping Range of Gold Nanoparticles. Nano Lett. 2005, 5, 1937-1942.

(32) Prikulis, J.; Svedberg, F.; Käll, M.; Enger, J.; Ramser, K.; Goksör, M.; Hanstorp, D. Optical Spectroscopy of Single Trapped Metal Nanoparticles in Solution. Nano Lett. 2004, 4, 115-118.

(33) Seol, Y.; Carpenter, A. E.; Perkins, T. T. Gold nanoparticles: enhanced optical trapping and sensitivity coupled with significant heating. Opt. Lett. 2006, 31, 2429-2431.

NL8015413 